\title{
Statistical association of discontinuities and reconnection in magnetohydrodynamic turbulence
}

S. Servidio, A. Greco

3 Dipartimento di Fisica, Università della Calabria, Italy

W. H. Matthaeus, K. T. Osman

4 Bartol Research Institute and Department of Physics and Astronomy,

${ }_{5}$ University of Delaware, USA

P. Dmitruk

- Departamento de Física, Universidad de Buenos Aires, Argentina

S. Servidio, Dipartimento di Fisica, Università della Calabria, Ponte P. Bucci, Cubo 31C, 87036 Cosenza, Italy (sergio.servidio@fis.unical.it)

A. Greco, Dipartimento di Fisica, Università della Calabria, Ponte P. Bucci, Cubo 31C, 87036 Cosenza, Italy (antonella.greco@fis.unical.it)

W. H. Matthaeus, Bartol Research Institute and Department of Physics and Astronomy, University of Delaware, Newark, DE 19716, USA (whm@udel.edu)

K. T. Osman, Bartol Research Institute and Department of Physics and Astronomy, University of Delaware, Newark, DE 19716, USA (kto@udel.edu)

P. Dmitruk, Departamento de Física, Facultad de Ciencias Exactas y Naturales, Universidad de Buenos Aires, Ciudad Universitaria, 1428, Buenos Aires, Argentina (pablo@bartol.udel.edu) 
X - 2 SERVIDIO ET AL.: DISCONTINUITIES AND RECONNECTION IN MHD TURBULENCE

7 Abstract. Using high Reynolds number simulations of two-dimensional

8 magnetohydrodynamic (2D MHD) turbulence as a test case, a statistical as-

9 sociation between tangential discontinuities and magnetic reconnection is demon-

${ }_{10}$ strated. Methods employed in previous studies on discontinuities and recon-

${ }_{11}$ nection in turbulence are used to identify sets of possible reconnection events

${ }_{12}$ along a one-dimensional path through the turbulent field, emulating exper-

13

imental sampling by single detector in a high speed flow. The goal is to de-

14

velop numerical algorithms for identifying candidate reconnection events in

15

space physics applications. We find that sets of strong discontinuities, iden-

tified using the normalized partial variance of vector increments, include an

increasing fraction of reconnection events as the threshold for identification grows. Magnetic discontinuities become almost purely reconnection events

for high thresholds, with values generally higher than six standard deviations. 


\section{Introduction}

Solar wind discontinuities are characterized by large and rapid changes in properties of the plasma and magnetic field [Burlaga, 1968; Tsurutani and Smith, 1979; Ness and Burlaga, 2001; Neugebauer, 2006; Vasquez et al., 2007]. Early surveys of solar wind discontinuities identified the majority as rotational discontinuities (Alfvén waves), but moremodern surveys identify most of them as tangential discontinuities (plasma boundaries). The time interval between the passages of subsequent strong (large-angle) discontinuities varies from seconds to hours. Thicknesses of strong discontinuities vary from $10^{3}-10^{5}$ $\mathrm{km}$.

One interpretation of the strong discontinuities is that they are the walls between filamentary structures of a discontinuous solar wind plasma [Burlaga, 1969; Borovsky, 2006; Borovsky and Denton, 2010], while another is that some strong discontinuities are fossils from the birth of the solar wind [Burlaga, 1968; Marsch and Tu, 1994; Borovsky, 2008]. Some could be formed away from the Sun by relaxation of the solar wind magnetic-field structure [Janse et al., 2010]. An alternative possibility is that the observed discontinuities are the current sheets that form as a consequence of the broad-band cascade of magnetohydrodynamic (MHD) turbulence [Matthaeus and Montgomery, 1980; Carbone et al., 1990]. Upon reaching kinetic scales, where the inertial range terminates in a medium such as the solar wind, one would expect numerous small-angle discontinuities with thickness as small as the ion inertial length or ion gyroradius (tens of kilometers), which are indeed observed [Vasquez et al., 2007]. Recent studies of inertial range indicators of discontinuities in the solar wind [Greco et al., 2008, 2009] show that their statistical distribution, 
41

and waiting time distribution, are very similar to distributions obtained from simulations of MHD turbulence.

In a traditional picture, solar wind fluctuations (e.g. Tu and Marsch [1993]; Neugebauer [2006]; Borovsky [2008]) are associated with classical convected (or propagating) MHD solutions. In that case, discontinuities are not viewed as an active ingredient of an evolving turbulent medium. How discontinuities evade such participation is not readily explained, except in linear small-amplitude theory. A parallel line of reasoning argues that thin current sheets are characteristic coherent structures expected in active intermittent MHD turbulence, and which are therefore integral to the dynamical couplings across scales. Such small scale dissipative coherent structures are candidates to be active sites of dissipation and magnetic reconnection [Matthaeus and Montgomery, 1980; Matthaeus and Lamkin , 1986]. Magnetic reconnection describes a process in which the MHD frozen-in field condition is violated in such a manner that magnetic field lines are reconfigured to produce topological changes in the field. This process generally favors oppositely directed magnetic fields at thin current sheets and plays a central role in many interpretations and models of space, solar, astrophysical, and laboratory plasma phenomena. Generally, magnetic reconnection has been studied in simplified geometries and boundary conditions, but since it might occur in any region separating topologically distinct magnetic flux structures, it might be of importance in more general circumstances, including near thin current sheets formed in MHD turbulence. The statistical properties of reconnection sites in two-dimensional (2D) MHD turbulence have been recently investigated, leading to the conclusion that strong reconnection events can locally occur in a turbulent magnetic field [Servidio et al., 2009, 2010]. 
64

In this perspective one is led naturally to suspect that at least some of the current sheets that are a common feature of the solar wind at $1 \mathrm{AU}$, may be participating in small-scale magnetic reconnection [Sundkvist et al., 2007], as well as inhomogeneous interplanetary plasma dissipation and heating [Leamon et al., 2000; Osman et al., 2011]. Two very important aspects of the latter chain of reasoning have not been established as far as we are aware. These are, first, to further establish the relationship between current sheets and small scale reconnection in turbulence. Some quantitative connection is needed. We have in mind the particular question: If one identifies a current sheet in turbulence, how likely is it to be also an active reconnection site? Second, frequent small scale reconnections have yet to be identified in the solar wind, although with use of higher resolution datasets the frequency of finding interplanetary reconnection has been increasing (see Gosling and Szabo [2008]; Phan et al. [2010]). It may be that an alternative approach would be useful, in which one can more or less automatically identify collections of events that are likely to be reconnection sites, and them look for detailed signatures as a subsequent step. Here we will contribute to both of these goals by showing, using MHD simulation data, that methods for identifying intermittent current sheet-like structures, when quantified properly, can identify sets of structures that are likely to be active reconnection regions.

Here we combine approaches, recently developed in studies of discontinuities and reconnection in turbulence, in order to identify reconnection events within a set of identified intermittent current events. We would prefer a more realistic 3D study, but we adopt a 2D approach to simplify the problem. Not only are much higher resolution simulations feasible in 2D, but also available schemes for identification of 3D reconnection properties are limited at present [Priest et al., 2003]. The 2D approximation, on the other hand, 
may not be so unreasonable as a first approximation of relevance to the solar wind. For example spectral anisotropy, of a type favoring 2D wave vectors, is a well known feature of MHD turbulence in the presence of a uniform mean magnetic field [Shebalin et al., 1983; Matthaeus et al., 1996]. Various studies (e.g., Matthaeus et al. [1990]; Bieber et al. $[1994,1996])$ also show that solar wind fluctuations appear to have, in effect, a large fraction of energy (maybe $80 \%$ or more) in quasi-2D or 2D wave vectors. Since a fully $3 \mathrm{D}$ numerical treatment of the type we undertake is unfeasible at present, we proceed using 2D MHD, which can attain higher spatial resolution.

The outline of the paper is as follows: In Sec. 2 the numerical simulations of turbulence are briefly reviewed. The techniques for the identification of discontinuities as reconnection events are described in Sec. 3. The local analysis of the topology of the discontinuities is investigated in Sec. 4, while an example of solar wind analysis is shown in Sec. 5. Finally, in Sec. 6, a summary and conclusions are given.

\section{Reconnection sites in $2 \mathrm{D}$ turbulence}

The 2D incompressible MHD equations can be written in terms of the magnetic potential $a(x, y)$ and a single component of vorticity $\omega(x, y)$, with uniform mass density $\rho=1$, as

$$
\begin{aligned}
& \frac{\partial \omega}{\partial t}=-(\boldsymbol{v} \cdot \nabla) \omega+(\boldsymbol{b} \cdot \boldsymbol{\nabla}) j+R_{\nu}^{-1} \nabla^{2} \omega, \\
& \frac{\partial a}{\partial t}=-(\boldsymbol{v} \cdot \nabla) a+R_{\mu}^{-1} \nabla^{2} a,
\end{aligned}
$$

where the velocity field $\boldsymbol{v}$ is solenoidal, $\nabla \cdot \boldsymbol{v}=0$, the magnetic field is $\boldsymbol{b}=\boldsymbol{\nabla} a \times \hat{\boldsymbol{z}}$, and the the electric current density is $j=-\nabla^{2} a$. The velocity $\boldsymbol{v}=\boldsymbol{\nabla} \phi \times \hat{\boldsymbol{z}}$ can be written in terms of a stream function $\phi$, related to the vorticity by $\omega=-\nabla^{2} \phi$. Eqs. (1)-(2) are written in familiar Alfvén units with lengths scaled to $L_{0}$, a typical large scale length. All quantities 
are in a set of dimensionless units defined by scaling velocities and magnetic fields to the root mean square Alfvén speed $C_{A}$, while time is scaled to $L_{0} / C_{A}$. The constant coefficients $R_{\mu}$ and $R_{\nu}$ are reciprocals of kinematic viscosity and resistivity, respectively, and represent magnetic and kinetic Reynolds numbers at scale $L_{0}$.

Eqs. (1)-(2) are solved in doubly periodic $(x, y)$ Cartesian geometry in a box of side $2 \pi L_{0}$, employing a dealiased (2/3 rule) pseudo-spectral code [Ghosh et al., 1993]. We employ a standard Laplacian dissipation with constant dissipation coefficients. In this work we show results from a run with $4096^{2}$ grid points and $R_{\nu}=R_{\mu}=1700$. Time integration is second order Runge-Kutta and double precision is employed. In the Fourier pseudospectral representation, the fluctuations are confined initially in the shell $4 \leq k \leq 10$, where the wavenumber $k$ is in units of $1 / L_{0}$. Mean values such as energy per unit mass, $E=1 / 2\left\langle|\boldsymbol{v}|^{2}+|\boldsymbol{b}|^{2}\right\rangle$, are expressed in terms of $\langle\bullet\rangle$ which denotes a spatial average. Random phases are employed for the initial Fourier coefficients, which are therefore initially uncorrelated. Initial velocity and magnetic field fluctuations are equipartitioned. With the above parameters, the turbulence correlation length is $\lambda_{C}=1.8 \times 10^{-1}$, and the dissipation length is $\lambda_{d}=4.6 \times 10^{-3}$.

For the present statistical analysis we will consider the state of the MHD system at $t \sim$ 0.4, when the peak of turbulent activity is achieved and the mean square current density $\left\langle j^{2}\right\rangle$ is near to its peak value. When the turbulence is fully developed, coherent structures appear. The large scale structures can be identified as magnetic islands that have different size and energy, and these are supported by cores of electric current density, in accord with Ampere's law. The dynamics are such that between the islands the perpendicular (outof-plane) component of the electric current density $j$ becomes very large, as reported in 
Fig. 1. These often very thin current structures are the small scale dynamically produced magnetic structures that are characteristic of 2D MHD - these are generally not present in random-phase initial data.

In previous studies [Servidio et al., 2009, 2010] it has been confirmed that turbulence leads to the spontaneous formation of local and intermittent reconnection events. We employed a cellular automata technique [Servidio et al., 2010], applied to the 2D turbulent field, permitting identification of the diffusion regions. First, the position of each magnetic X-point (saddle point of potential $a$ ) is found. Second, at these points, the width $d$, the elongation $\ell$, and the respective reconnection rate $E_{\times}$are systematically computed making use of the Hessian of the magnetic potential a [Servidio et al., 2010]. Third, the threshold (edge condition) for the cellular automata is chosen to be the value of the current at $\pm d / 2$, centered at the X-point. Finally, an index $n$ is used to identify each island. The cellular automaton is designed to propagate the index $n$ away from the peak of the current located near the nth X-point, towards the local edge condition on the current, where propagation of the index ceases. With the above procedure the shape and the position of each diffusion region is defined. The reconnection rate associated with a particular reconnection region is just the electric field evaluated at the magnetic neutral point [Servidio et al., 2009, 2010].

A full analysis of the above type is carried out for each of the strong reconnection sites [Servidio et al., 2010], defined in the following way. The set containing all the strongest reconnection sites (Total Reconnection Sites, designated as TRS) can be identified by comparing the dynamical result from the MHD simulation with analysis of another magnetic field obtained from the first by holding the magnetic spectrum constant and randomizing the phases of the Fourier coefficients. This "Gaussianization" procedure actually 
increases the number of X-points, but effectively eliminates the strongest reconnection rates. The approximate boundary between the Gaussianized distribution of rates and the "tail" of strong rates is a natural way to differentiate between "strong" and "weak" reconnection sites for a given turbulence snapshot. See Servidio et al. [2010] for details. The strong reconnection sites defined in this way are spatially mapped using the above cellular automata procedure. The set of strong reconnection sites $T R S$, having number of elements \#TRS, and their associated surrounding reconnection regions, forms the basis of the results shown in the next session.

\section{Discontinuities and reconnection events}

The main purpose of the present investigation is to identify a possible link between bursty reconnection sites and tangential discontinuities (TDs). Anticipating possible applications to spacecraft data, we focus on properties of discontinuities that are recorded by magnetic field measurements at a single spacecraft in interplanetary space. In order to establish such a link, we adopt a spacecraft-like sampling through the simulation domain (see e.g Greco et al. [2008]), as discussed below. We fix our attention on one specific trajectory and the properties it samples. In particular, we can define a set whose elements consist of the segments of a trajectory that passes through any reconnection zone, identified by the cellular automaton method. In this way we can build a set of strong reconnection site encounters $R S$ associated with a trajectory. Let us denote the number of strong reconnection site encounters found along the trajectory by \#RS. Note that $R S$ is not strictly a subset of $T R S$, i.e., we cannot conclude strictly that $R S \subseteq T R S$. To see this recall that $T R S$ is a set of regions or areas, and $R S$ is a set of segments of a trajectory. A trajectory may sample a particular reconnection region once, not at all, or more 
than once. To the extent that the last possibility is rare, we expect that \#RS $\leq \# T R S$. However, it is also possible that a particular reconnection region contributes more than one element to the set $R S$ if the trajectory crosses the reconnection region twice or more. This will not cause any additional difficulty below because we are mainly interested in $R S$, the set of encounters with reconnection regions along the trajectory.

To simulate spacecraft measurements of the magnetic field along the selected trajectory, we adopt the same technique used by Greco et al. [2008], but here arranged for the 2D case. We interpolate the data along the selected path through the simulation box, and on these data we identify TDs using a statistical method, as follows: First, to describe rapid changes in the magnetic field, we look at the increments

$$
\Delta \mathbf{b}(s, \Delta s)=\mathbf{b}(s+\Delta s)-\mathbf{b}(s),
$$

where $\boldsymbol{b}$ the magnetic field, $s$ the 1D coordinate along the trajectory, and $\Delta s$ the spatial separation or lag. For this simulation we choose a small scale lag, $\Delta s \simeq 0.67 \lambda_{d}$, which is comparable to $\lambda_{d}$, the turbulence dissipation length.

Employing only the sequence of magnetic increments, we compute the normalized magnitude

$$
\Im(\Delta s, \ell, s)=\frac{|\Delta \mathbf{b}(s, \Delta s)|}{\sqrt{\left\langle|\Delta \boldsymbol{b}(s, \Delta s)|^{2}\right\rangle_{\ell}}},
$$

where $\langle\bullet\rangle_{\ell}=(1 / \ell) \int_{\ell} \bullet d s$ denotes a spatial average over an interval of length $\ell$, and $\Delta s$ is the spatial lag in Eq. (3). The square of the above quantity has been called the Partial Variance of Increments (PVI) [Greco et al., 2008] and the method abbreviated as the PVI method.

For the numerical analysis performed here $\ell \simeq 535 \lambda_{C}$, where $\lambda_{C}=0.18$ is the turbulence correlation length - a natural scale for computing averages. The choice of $\ell$ as a 


\subsection{Effects of thresholds on recognition of reconnection sites}

Imposing a threshold $\theta$ on Eq. (4), a collection of stronger discontinuities along the path $s$ can be identified. That is, we select portions of the trajectory in which the condition

$$
\Im(\Delta s, \ell, s)>\theta
$$

is satisfied, and we will employ this condition to identify candidate reconnection sites.

In Fig. 3, an example of the location of discontinuities along s, selected by the PVI method with a particular threshold $\theta$, is shown. The figure also shows reconnection sites identified using the cellular automaton method described earlier. One can immediately see in Fig. 3 that there is an association, but not an identity, between the set of "events" identified using Eq. (5), and the encounters of the trajectory with reconnection regions. We will now study this association quantitatively using different values of threshold $\theta$. 
X - 12 SERVIDIO ET AL.: DISCONTINUITIES AND RECONNECTION IN MHD TURBULENCE

To evaluate possible refinements to the approach, we include in some tests an additional threshold condition, namely that

$$
|\mathbf{b}|<\beta
$$
there are 37 reconnection sites along the path $s$, that is $\# R S=37$. strong reconnection, and therefore are not of interest in this analysis.

are also reconnection sites (i.e., elements of the set $R S$ ), as follows: Every discontinuity is characterized by a starting and an ending point along the synthetic trajectory $s$. A set of discontinuities is identified, and a certain number of these discontinuities intersect reconnection regions. To automate the determination of the reconnection regions, we make use of a map [Servidio et al., 2010] that is generated using the cellular automaton procedure. The latter, in summary, is a $2 \mathrm{D}$ matrix that has 0 values in all cells outside of the diffusion regions, or values of 1 inside the diffusion regions. Reconnection regions are (blue) shaded in Fig. 3. For this simulation, and for the selected trajectory (see Fig. 3),

When at least one point of the identified candidate discontinuity overlaps with one point of the identified reconnection region, the event is counted as a "success". Otherwise the TD is not identified as an RS, and is a "failure". In the latter case the method is detecting a non-reconnecting, high-stress, magnetic field structure. Such regions may be physically interesting for other reasons. However, such points are not associated with a region of

To summarize, for a given threshold $\theta$ [see Eqs. (4) and (5)], there will be a set of identified TDs (a set ITD with number of elements \#ITD); there will be a subset of 
these discontinuities that are Identified Reconnection Sites (a set IRS with number of elements \#IRS). There will also be a complementary set of identified TDs that are not RSs, numbering \#ITD - \#IRS, and reconnection regions lying along the trajectory that are not captured at all by the technique, numbering \#RS - \#IRS.

As an example, using $\theta=5$ in Eq. (5), 40 discontinuities have been identified and 23 overlap a reconnection site and correspond to successful identification of a reconnection region. That is, \#ITD $=40$ and \#IRS $=23$. The goodness (quality) of this method can be defined as \#IRS/\#ITD, the number of the successes over the total number of identified discontinuities. For this example, the goodness is $\simeq 57.5 \%$. An example of discontinuities, together with the reconnecting regions, is shown in Fig. 3.

Following the above procedure summarized by Eqs. (5)-(6), we impose different thresholds $\theta$ and $\beta$ for the PVI signal. Each threshold characterizes a different set of discontinuities or "events", and we can label each algorithm as $\Im_{\theta}^{b<\beta}$. The parameters of different PVI-based algorithms are listed in Table 1 , all of which use $\Im\left(\Delta s=0.76 \lambda_{d}, \ell=535 \lambda_{C}\right)$. It can be seen that for higher values of $\theta$ an increasing fraction of the identified TDs corresponds to a reconnection site. That is, the goodness ratio \#IRS/\#ITD increases as the threshold $\theta$ is increased. It is clear from the Table that the goodness does not improve significantly by also imposing a threshold on field strength Eq. (6).

The performance of the family of methods, summarized in Table 1, is illustrated in Fig. 4. Here the variation of the the number of candidate events with varying threshold is shown by plotting the ratio $\# \operatorname{IT} D(\theta) / \# \operatorname{IT} D(\theta=1)$. This number decreases as a function of $\theta$, but slowly after $\theta>7$, beyond which there are less than $5 \%$ as many events as there are with the lowest threshold that we employed, $\theta=1$. We also show in the same 
X - 14 SERVIDIO ET AL.: DISCONTINUITIES AND RECONNECTION IN MHD TURBULENCE

plot the ratio of identified reconnection sites to identified discontinuities, \#IRS/\#ITD.

This quantity approaches $100 \%$ for the largest values of $\theta$ that we used. The efficiency, defined as the ratio of the number of identified reconnection sites \#IRS over the total number of reconnection regions along the path $\# R S$, is also represented, on the same plot, as a function of threshold.

Note that among the 378 TDs found with $\theta=1$, one finds that every one of the strong reconnection sites, 37 in number, actually lies along the trajectory. In this sense, a lower threshold such as $\theta=1$ is very effective - all reconnection sites are found. There are however a large number of false positives. In contrast, a large threshold value $(\theta=7$ or $\theta=8$ ) finds essentially only strong reconnection sites and has very few or no false positives, but identifies only about $1 / 3$ of the actual number of strong reconnection sites that are known to lie along the trajectory.

Recalling again that the method for identifying RSs was designed to only find stronger sites, we computed the distribution of the all the reconnection rates from the $2 \mathrm{D}$ field as the values of X-point electric field $\left|E_{\times}\right|$. See the references Servidio et al. [2009, 2010] for more details. This distribution function (PDF) is reported in Fig. 5-(a) and includes all reconnection sites, both strong and weak, in the entire 2D volume. We compare this distribution with the distribution of reconnection rates for the set of Identified Reconnection Sites found by one of the PVI-based cases. We choose the case with threshold $\theta=7.3$, because in this case all the identified discontinuities correspond to reconnection sites (see Table 1). The comparison of these reconnection rate PDFs is shown in Fig. 5. Evidently, the PVI techniques outlined above are capable of identifying the fastest and strongest reconnecting sites, namely those with higher reconnection rate $\left|E_{\times}\right|$. 


\subsection{Varying PVI averaging}

In this section we will compute the PVI series given in Eq. (4), varying the interval of averaging in the denominator,

$$
\Im(\Delta s, \ell, s)=\frac{|\Delta \mathbf{b}(s, \Delta s)|}{\sqrt{\frac{1}{\ell} \int_{s-\frac{\ell}{2}}^{s+\frac{\ell}{2}}}|\Delta \boldsymbol{b}(z, \Delta s)|^{2} d z} .
$$




\section{Local description of TD}

$$
\begin{aligned}
W(\Delta s, \ell, s) & =\frac{\left|\Delta_{2} \boldsymbol{b}\right|}{\left\langle\left|\Delta_{2} \boldsymbol{b}\right|^{2}\right\rangle_{\ell}}, \text { where } \\
\left|\Delta_{2} b_{j}\right| & =b_{j}(s-\Delta s)-2 b_{j}(s)+b_{j}(s+\Delta s),
\end{aligned}
$$

In this section we will provide some numerical algorithms that can be used for the local description of tangential discontinuities. In Sec. 3 we found that for higher threshold $\theta$ the number of identified discontinuities \#ITD is equal to the number of reconnection sites \#RS along the path (goodness $=100 \%$ ), so here we will analyze the series obtained using $\Im_{8}\left(\Delta s=0.67 \lambda_{d}, \ell=535 \lambda_{c}\right)$, where $\theta=8$. In this case $\# I T D=\# R S=13$, as reported in Table 1. An example of a TD (or RS) is reported in Fig. 8-(a). The shaded (blue) region represents the limits along the trajectory of the reconnection zone, as captured by the cellular automata technique. As it can be seen, a very rough estimation of the width of the TD is size of region (interval of $s$ ) in which the PVI signal exceeds the selected threshold. However, this definition is not very precise because it depends not only on the amplitude of $\Im$ nearby the TD, and on $\theta$, but also upon the angle with which the trajectory encounters the discontinuity. In order to avoid this problem we will use as the (optimized) width $\delta$ of the TD the distance between two peaks in another signal, namely, 
SERVIDIO ET AL.: DISCONTINUITIES AND RECONNECTION IN MHD TURBUlENCE X - 17

$$
S_{i j}=\left\langle b_{i} b_{j}\right\rangle-\left\langle b_{i}\right\rangle\left\langle b_{j}\right\rangle,
$$


$\mathrm{X}-18$

where here $\langle\ldots\rangle$ denotes an average on the trajectory within the TD. Then we compute the eigenvalues $\left(\lambda_{1}, \lambda_{2}\right)$ and the normalized eigenvectors $(\hat{\mathbf{n}}, \hat{\mathbf{t}})$, where $\lambda_{1}$ is the maximum eigenvalue and $\hat{\mathbf{n}}(\hat{\mathbf{t}})$ is the normal (tangential) eigenvector. The system of eigenvectors, for the same TD represented in Fig. 8, is shown in Fig. 9. The values of the ratio $\lambda_{1} / \lambda_{2}$ is very large for all the discontinuities selected by $\Im_{8}$, that is $100<\lambda_{1} / \lambda_{2}<10^{7}$. Another feature is that the normal component $b_{n}$ is almost null and constant, while $b_{t}$ is strongly changing sign (as is typical of models of reconnection.) The normal and tangential magnetic field in a sample IRS is reported in Fig. 9.

As was previously pointed out by Erdos and Balogh [2008], if the normal vector to the discontinuity $\hat{\mathbf{n}}$ is not aligned with the flow direction (in this case the trajectory direction $\hat{\mathbf{s}})$, then the width $\delta$ is overestimated. For each TD captured by $\Im$, using the $W$-field we measured each $\delta$, and we optimized each one with $\delta^{\prime}=\delta \cos \alpha$, where $\alpha$ is the angle between $\hat{\mathbf{n}}$ and $\hat{\mathbf{s}}$. For this simulation, taking an average on all the discontinuities, we obtained $\langle\delta\rangle=1.68 \times 10^{-2}$ and $\left\langle\delta^{\prime}\right\rangle=1.45 \times 10^{-2}$. From the $2 \mathrm{D}$ simulation we measured for each reconnection site the width of the diffusion region $d$ using the method described in Servidio et al. [2010] and we obtained that $\langle d\rangle=1.40 \times 10^{-2}$. The estimation $\left\langle\delta^{\prime}\right\rangle$ is in very good agreement with the average size of the diffusion region $\langle d\rangle$.

\section{An example from solar wind}

We have computed the PVI time series using ACE 1 second resolution magnetic field data from the interval 2004 May 1 to 18 . The increment $(\Delta s)$ is 20 seconds and the averaging interval in the denominator in Eq. (4) is the entire data period. The average velocity was around $400 \mathrm{~km} / \mathrm{s}$. In Fig. 10, the PVI time series is shown. In order to facilitate the comparison with Fig. 2, we converted the time signal to a spatial signal, 
$\mathrm{X}-20$ SERVIDIO ET AL.: DISCONTINUITIES AND RECONNECTION IN MHD TURBULENCE

reconnection sites. Once the RS are identified, we also perform a local analysis in order to describe the topology of the reconnection sites. The algorithm has been tested with very good results on the 2D simulations. We are presently working on an implementation of the present methods for solar wind data, extending beyond the simple example provided in the previous Section 5. We anticipate that an interesting collection of discontinuities can be identified and then examined in detail to test against reported identifications of reconnection sites using other methods [Gosling and Szabo, 2008; Phan et al., 2010]. It may be possible using this approach to uncover numerous reconnection events that might be present in the solar wind, but have not yet been identified. In general we suggest that the methods developed here may be useful for identification of candidate reconnection sites from magnetic field data obtained in a turbulent plasma. We are also studying extensions to three dimensions, which we expect to be difficult (see, e.g., Priest et al. [2003]).

Acknowledgments. This research supported in part by the NASA Heliophysics Theory Program (NNX08AI47G); the MMS project (NNX08AT76G); the NASA Solar Probe Plus ISIS team, the NASA Geospace Sciences (NNX08AM48G), and the NSF SHINE program (ATM-0752135). S. S. would like to acknowledge the grant "Giovani Ricercatori2010" at UNICAL. 


\section{References}

Bieber, J. W., W. H. Matthaeus, C. W. Smith, W. Wanner, M.-B. Kallenrode, and G. Wibberenz (1994), Proton and electron mean free paths: The Palmer consensus revisited, Astrophys. J., 420, 294.

Bieber, J. W., W. Wanner, and W. H. Matthaeus, (1996), Dominant two-dimensional solar wind turbulence with implications for cosmic ray transport, J. Geophys. Res., $101,2511$.

Borovsky, J. (2006), Eddy viscosity and flow properties of the solar wind: Co-rotating interaction regions, coronal-mass-ejection sheaths, and solar-wind/magnetosphere coupling, Phys. Plasmas, 13, 056505.

Borovsky, J. (2008), Flux tube texture of the solar wind: Strands of the magnetic carpet at 1 AU?, J. Geophys. Res., 113, 08110.

Borovsky, J. and M. H. Denton (2010), Solar wind turbulence and shear: A superposedepoch analysis of corotating interaction regions at 1 AU, J. Geophys. Res., 115, 10101.

Burlaga, L. F.(1968), Micro-Scale Structures in the Interplanetary Medium, Solar Physics, $4,67$.

Burlaga, L. F. (1969), Directional Discontinuities in the Interplanetary Magnetic Field, Solar Physics, \%, 54.

Carbone, V., P. Veltri, and A. Mangeney (1990), Coherent structure formation and magnetic field line reconnection in magnetohydrodynamic turbulence, Physics of Fluids, 2, 1487 .

Erdos, G. and A. Balogh (2008), Density of discontinuities in the heliosphere, Adv. Space Res., 41, 287. 
X - 22 SERVIDIO ET AL.: DISCONTINUITIES AND RECONNECTION IN MHD TURBULENCE

Ghosh, S., M. Hossain, and W. H. Matthaeus (1993), The application of spectral methods in simulating compressible fluid and magnetofluid turbulence, Comput. Phys. Commun., $74,18$.

Gosling, J. T. and A. Szabo (2008), Bifurcated current sheets produced by magnetic reconnection in the solar wind, J. Geophys. Res., 113, A10103.

Greco, A., P. Chuychai, W. H. Matthaeus, S. Servidio, and P. Dmitruk (2008), Intermittent MHD structures and classical discontinuities, Geophys. Res. Lett., 35, L19111.

Greco, A., W. H. Matthaeus, S. Servidio, P. Chuychai, P. Dmitruk (2009), Statistical Analysis of Discontinuities in Solar Wind ACE Data and Comparison with Intermittent MHD Turbulence, Astrophys. J., 691, L111 .

Janse, A. M. , B. C. Low, and E. N. Parker (2010), Topological complexity and tangential discontinuity in magnetic fields, Phys. Plasmas, 17, 092901.

Leamon, R. J., W. H. Matthaeus, C. W. Smith, , G. P. Zank, D. J. Mullan, and S. Oughton (2000), MHD-driven Kinetic Dissipation in the Solar Wind and Corona, Astrophys. J., $537,1054$.

Marsch, E. and C. Y. Tu (1994), Non-Gaussian probability distributions of solar wind fluctuations, Annales Geophysicae, 12, 1127.

Matthaeus, W. H., and D. Montgomery (1980), Selective decay hypothesis at high mechanical and magnetic Reynolds numbers, Annals of the New York Academy of Sciences , 357, 203.

Matthaeus, W. H., and S. L. Lamkin, (1986) Turbulent Magnetic Reconnection, Phys. Fluids, 29, 2513. 
Matthaeus, W. H., M. L. Goldstein, and D. A. Roberts (1990), Evidence for the presence of quasi-two-dimensional nearly incompressible fluctuations in the solar wind, J. Geophys. Res., 95, 20673.

Matthaeus, W. H. , S. Ghosh, S. Oughton, and D. A. Roberts (1996), Anisotropic threedimensional MHD turbulence, J. Geophys. Res., 101, 7619.

Matthaeus, W. H. , S. Dasso, J. M. Weygand, L. J. Milano, C. W. Smith, and M. G. Kivelson (2005), Spatial Correlation of Solar-Wind Turbulence from Two-Point Measurements, Phys. Rev. Lett., 95, 231101.

Ness, N. F. , and L. F. Burlaga (2001), Spacecraft studies of the interplanetary magnetic field, J. Geophys. Res., 106, 15803.

Neugebauer, M. (2006), Comment on the abundances of rotational and tangential discontinuities in the solar wind, J. Geophys. Res., 111, A04103.

Osman, K. T., W. H. Matthaeus, A. Greco, and S. Servidio (2011), Evidence for Inhomogeneous Heating in the Solar Wind, Astrophys. J., 727, L11.

Perri, S., E. Yordanova, V. Carbone, P. Veltri, L. Sorriso-Valvo, R. Bruno, and M. André (2009), Magnetic turbulence in space plasmas: Scale-dependent effects of anisotropy, J. Geophys. Res., 114, A02102.

Phan, T. D., J. T. Gosling, G. Paschmann, C. Pasma, J. F. Drake, M. Oieroset, D. Larson, R. P. Lin, and M. S. Davis (2010), The Dependence of Magnetic Reconnection on Plasma and Magnetic Shear: Evidence from Solar Wind Observations, Astrophys. J., 719, L199.

Priest, E. R. , G. Hornig, and D. I. Pontin (2003), On the nature of three-dimensional magnetic reconnection, J. Geophys. Res., 108, 1285. 
X - 24 SERVIDIO ET AL.: DISCONTINUITIES AND RECONNECTION IN MHD TURBULENCE

Servidio, S., W. H. Matthaeus, M. A. Shay, P. A. Cassak and P. Dmitruk (2009), Magnetic Reconnection in Two-Dimensional Magnetohydrodynamic Turbulence, Phys. Rev. Lett., 102, 115003.

Servidio, S. , W. H. Matthaeus, M. A. Shay, P. Dmitruk, P. A. Cassak, and M. Wan (2010), Statistics of magnetic reconnection in two-dimensional magnetohydrodynamic turbulence, Phys. Plasmas, 17, 032315.

Shebalin, J. V. , W. H. Matthaeus, and D. Montgomery (1983), Anisotropy in MHD turbulence due to a mean magnetic field, J. Plasma Phys., 29, 525.

Sonnerup, B. U. O. , and L. J. Cahill (1967), Magnetopause Structure and Attitude from Explorer 12 Observations, J. Geophys. Res., 72, 171.

Sundkvist, D. , A. Retino, A. Vaivads A., and S. D. Bale (2007), Dissipation in Turbulent Plasma due to Reconnection in Thin Current Sheets, Phys. Rev. Lett. , 99, 025004.

Tsurutani, B. T. , and E. J. Smith (1979), Interplanetary discontinuities - Temporal variations and the radial gradient from 1 to 8.5 AU, J. Geophys. Res., 84, 2773.

Tu, C.-Y., and E. Marsch (1993), A model of solar wind fluctuations with two components - Alfven waves and convective structures , J. Geophys. Res., 98, 1257.

Vasquez, B. J. , V. I. Abramenko, D. K. Haggerty, and C. W. Smith (2007), Numerous small magnetic field discontinuities of Bartels rotation 2286 and the potential role of Alfvnic turbulence, J. Geophys. Res., 112, 11102.

Veltri, P. (1994), Low frequency turbulence and energy dissipation in the Solar Wind, Space Science Reviews, 68, 63. 


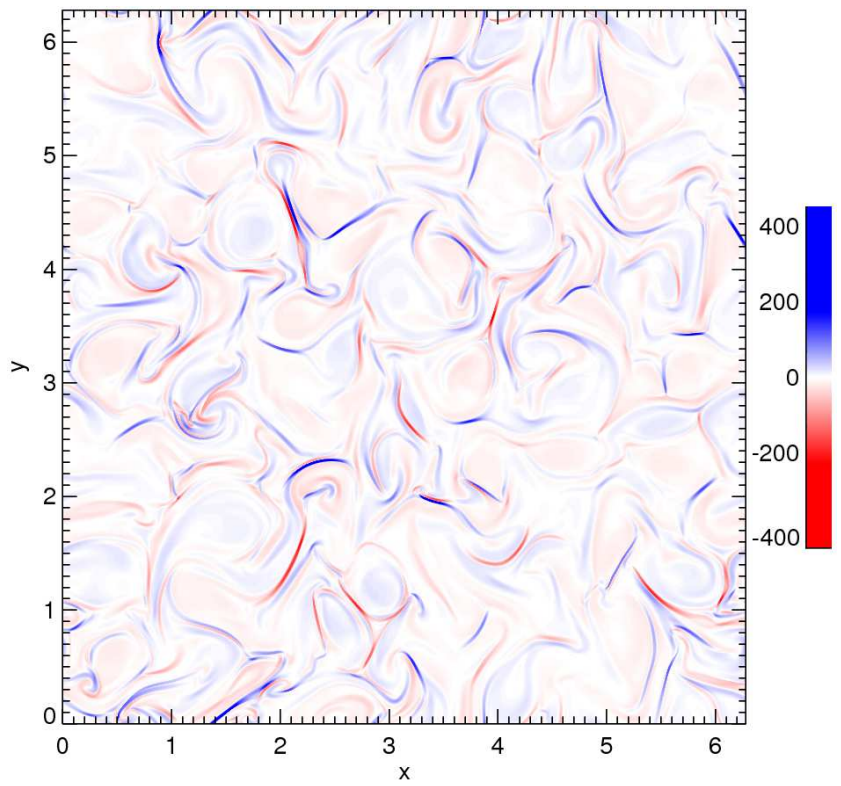

Figure 1. A contour map of the out-of-plane current density for the MHD simulation used in the present analysis.

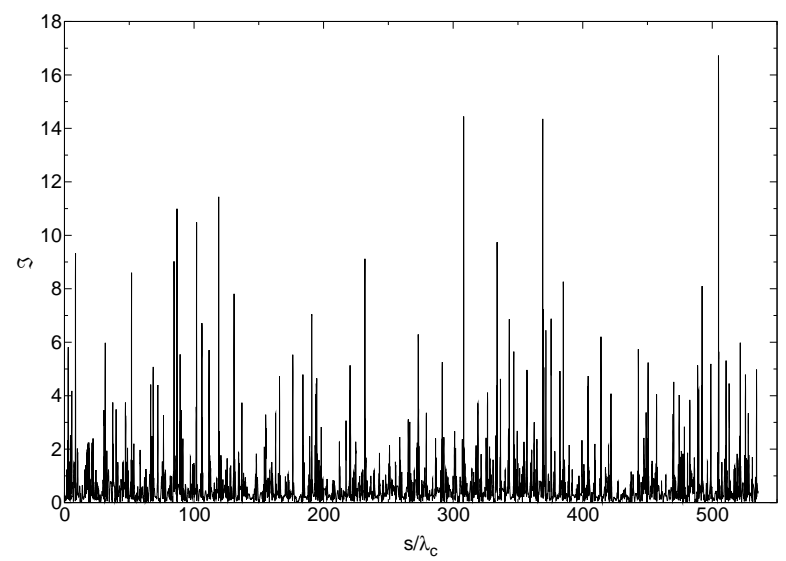

Figure 2. Spatial signal $\Im(\Delta s, \ell, s)$ (PVI) obtained from the simulation by sampling along the trajectory $s$ in the simulation box, with $\Delta s \simeq 0.67 \lambda_{d}$ and $\ell \simeq 535 \lambda_{C}$. This spatial signal can be compared to a time signal measured by solar wind spacecraft. 


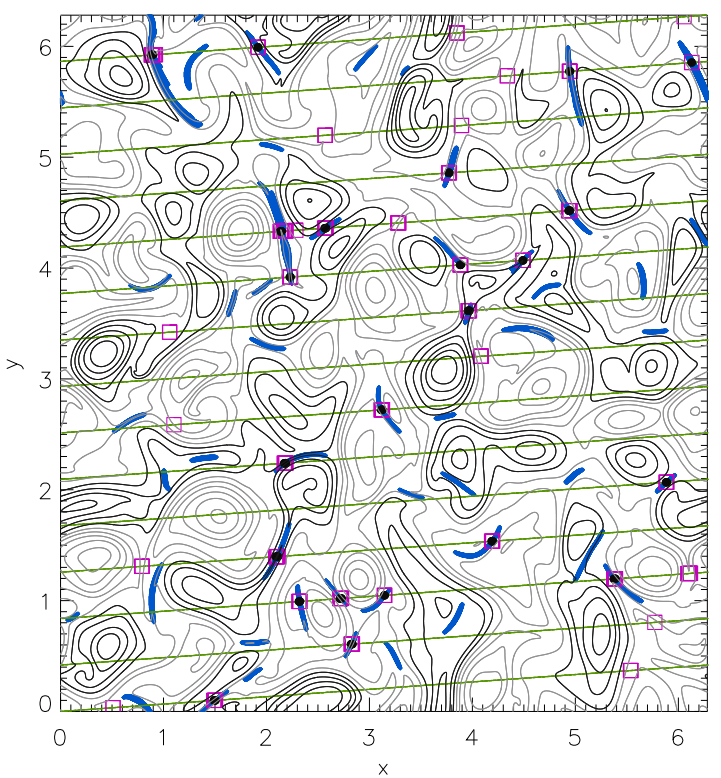

Figure 3. Contour lines of the magnetic field (or line contour of $a$ ) together with the diffusion regions (blue shaded map), and with the one-dimensional path $s$ (green solid line). On the same plot, the discontinuities identified by PVI technique with a threshold $\theta=5$ in Eq. (4) (open magenta squares) are represented. Bullets (black) are discontinuities which correspond to reconnection sites. The goodness of the technique is proportional to the ratio between the total number of identified TDs and the known RSs, i.e., \#IRS/\#ITD. 


\begin{tabular}{|c|c|c|c|c|c|c|}
\hline Method & $\theta$ & $\beta$ & \# ITD & \# IRS & efficiency (\%) & goodness (\%) \\
\hline$\Im_{1}$ & 1 & $/$ & 378 & 37 & 100 & 9.8 \\
$\Im_{2}$ & 2 & $/$ & 152 & 35 & 94.6 & 23.0 \\
$\Im_{3}$ & 3 & $/$ & 89 & 31 & 83.8 & 34.8 \\
$\Im_{4}$ & 4 & $/$ & 64 & 28 & 75.7 & 43.7 \\
$\Im_{5}$ & 5 & $/$ & 40 & 23 & 62.2 & 57.5 \\
$\Im_{6}$ & 6 & $/$ & 25 & 18 & 48.6 & 72.0 \\
$\Im_{7}$ & 7 & $/$ & 16 & 15 & 40.5 & 93.7 \\
$\Im_{7.3}$ & 7.3 & $/$ & 14 & 14 & 37.8 & 100 \\
$\Im_{8}$ & 8 & $/$ & 13 & 13 & 35.1 & 100 \\
$\Im_{1}^{b<1}$ & 1 & 1 & 295 & 37 & 100 & 12.5 \\
$\Im_{4}^{b<1}$ & 4 & 1 & 57 & 26 & 70.3 & 45.6 \\
$\Im_{7}^{b<1}$ & 7 & 1 & 16 & 15 & 40.5 & 93.7 \\
$\Im_{1}^{b<0.3}$ & 1 & 0.3 & 163 & 31 & 83.8 & 19 \\
$\Im_{4}^{b}<0.3$ & 4 & 0.3 & 44 & 24 & 64.9 & 54.5 \\
$\Im_{7}^{b<0.3}$ & 7 & 0.3 & 13 & 12 & 32.4 & 92.3 \\
\hline
\end{tabular}

Table 1. First column: label of the method $\Im_{\theta}^{b<\beta}$. Second column: threshold $\theta$ imposed on PVI, cf., Eq.(5). The character "/" indicates that no restriction is imposed. Third column: the value of the threshold $\beta$ on $|\boldsymbol{b}|$ Eq. (6). Fourth column: \#ITD, number of discontinuities identified by the method. Fifth column: \#IRS, number of reconnection sites found by the method. Sixth column: \#IRS/\#RS, the relative efficiency of the method, identified reconnection sites as percent of all the reconnection sites present along the path. Last column: \#IRS/\#ITD, the relative goodness of the method, percent of identified reconnection events in set of identified discontinuities.

\begin{tabular}{|c|c|c|c|c|c|c|}
\hline Method & $\theta$ & $\beta$ & \# ITD & \# IRS & efficiency (\%) & goodness (\%) \\
\hline$\Im_{1}$ & 1 & $/$ & 520 & 37 & 100 & 7.1 \\
$\Im_{2}$ & 2 & $/$ & 171 & 35 & 94.6 & 20.5 \\
$\Im_{3}$ & 3 & $/$ & 61 & 28 & 75.7 & 45.9 \\
$\Im_{4}$ & 4 & $/$ & 27 & 16 & 43.2 & 59.2 \\
$\Im_{4.5}$ & 4.5 & $/$ & 7 & 6 & 16.2 & 85.7 \\
$\Im_{5}$ & 5 & $/$ & 1 & 1 & 2.7 & 100 \\
\hline
\end{tabular}

Table 2. Same format of Table 1, but from the events found using a PVI series $\Im\left(\Delta s=0.67 \lambda_{d}, \ell=2 \lambda_{C}\right)$. 


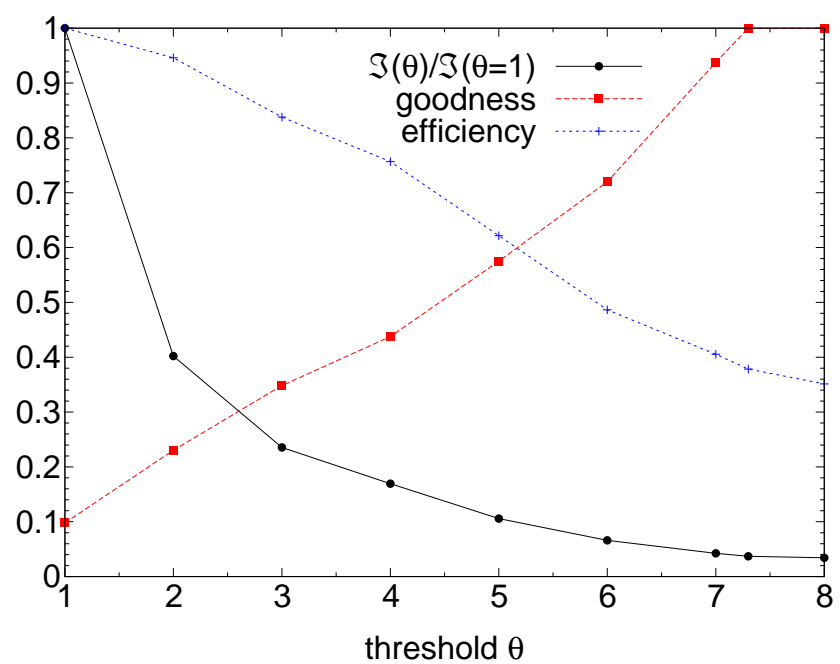

Figure 4. $\# I T D(\theta) / \# I T D(\theta=1)$, number of identified TD events as a function of the threshold $\theta$, normalized by the value at $\theta=1$ (black bullets). Also shown as a function of $\theta$ are the ratio of identified reconnection sites to total identified discontinuity "events" for threshold $\theta$, \#IRS/\#ITD (i.e., goodness, full red squares), and the ratio of identified reconnection sites to total reconnection sites along the trajectory, \#IRS( $\theta) / \# R S$ (the efficiency, blue crosses). All these methods use the PVI series defined by $\Im\left(\Delta s=0.76 \lambda_{d}, \ell=535 \lambda_{C}\right)$. 

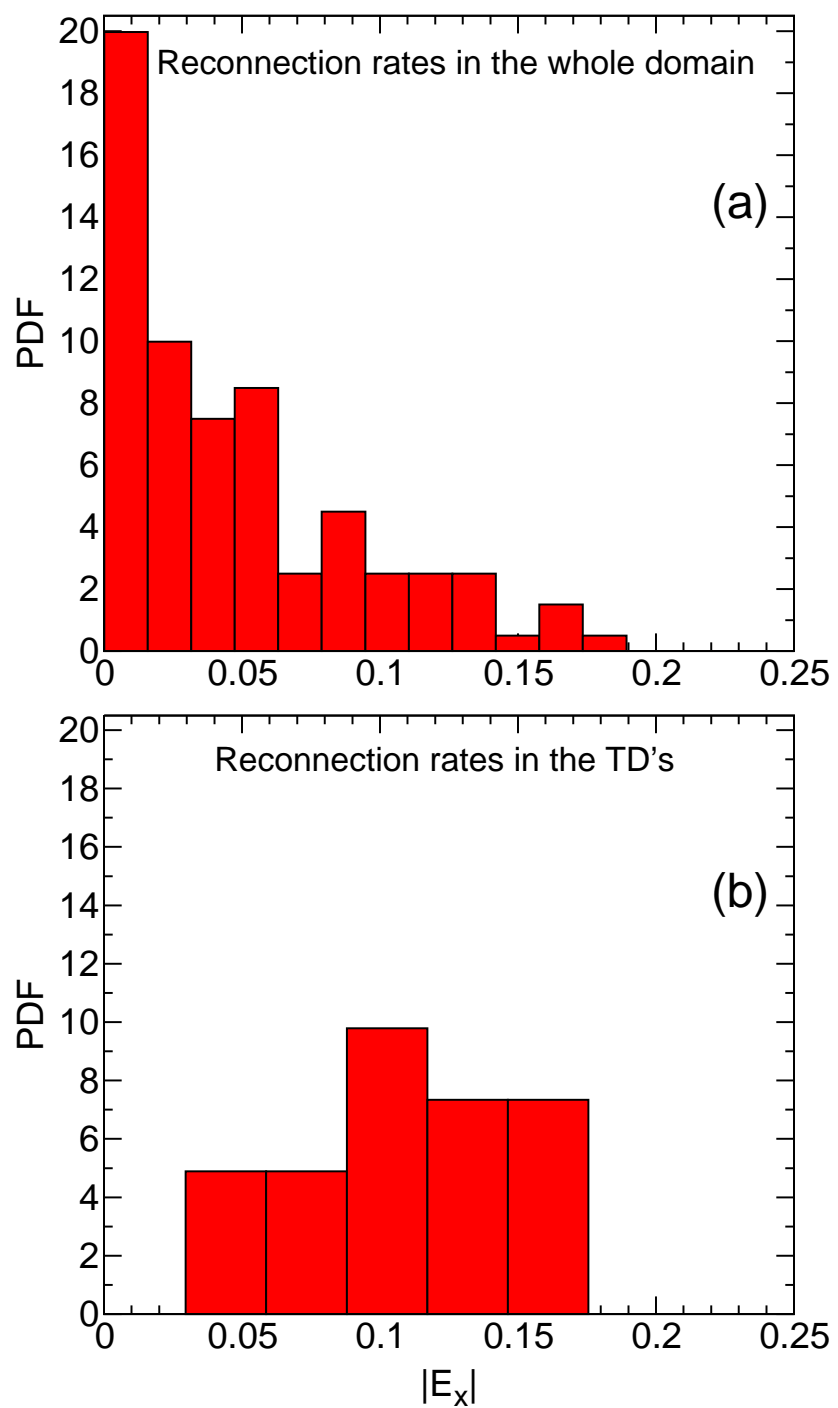

Figure 5. Comparison between the distribution of the reconnection rates. Panel (a): reconnection rates for all the reconnection sites - weak and strong. Panel (b): reconnection rates in the TDs, identified by $\Im\left(\Delta s=0.67 \lambda_{d}, \ell=535 \lambda_{C}\right)$, that are RSs (threshold $\theta=7.3$ ). Note that the cellular automata algorithm captures only RS with $\left|E_{\times}\right|>0.015$. 


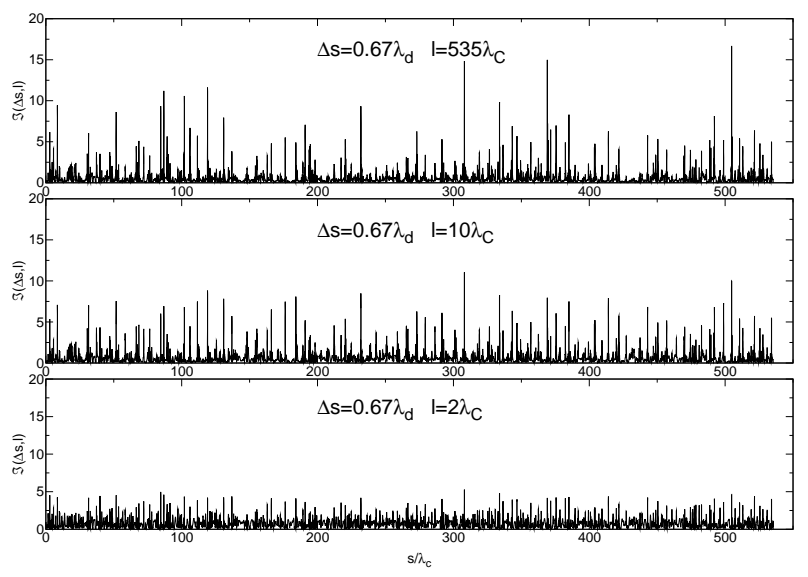

Figure 6. PVI-series computed from Eq. (7), using $\ell=535 \lambda_{C}$ (top), $\ell=10 \lambda_{C}$ (middle) and $\ell=2 \lambda_{C}$ (bottom). Going to small $\ell$, the signal becomes weaker.

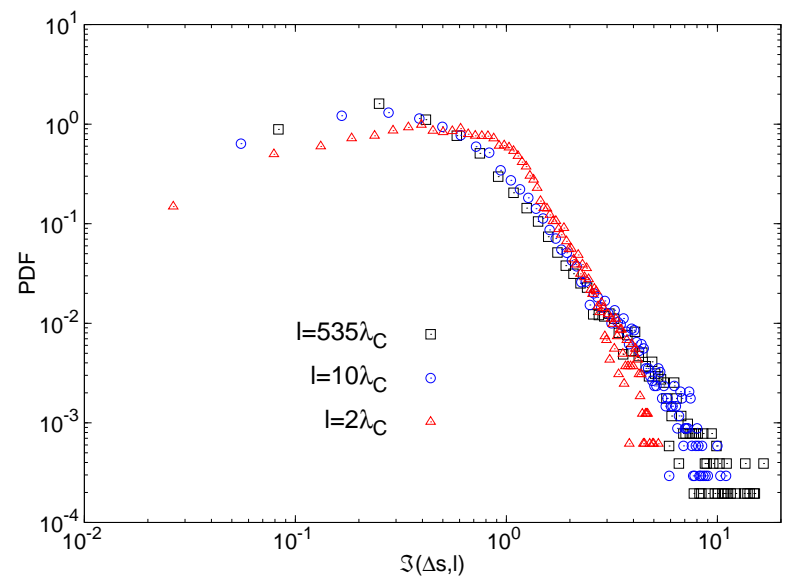

Figure 7. $\quad$ PDF of the pvi-series computed from Eq. (7), using $\ell=535 \lambda_{C}$ (black squares), $\ell=10 \lambda_{C}$ (blue circles) and $\ell=2 \lambda_{C}$ (red triangles). Diminishing $\ell$ the signal becomes less intermittent. 

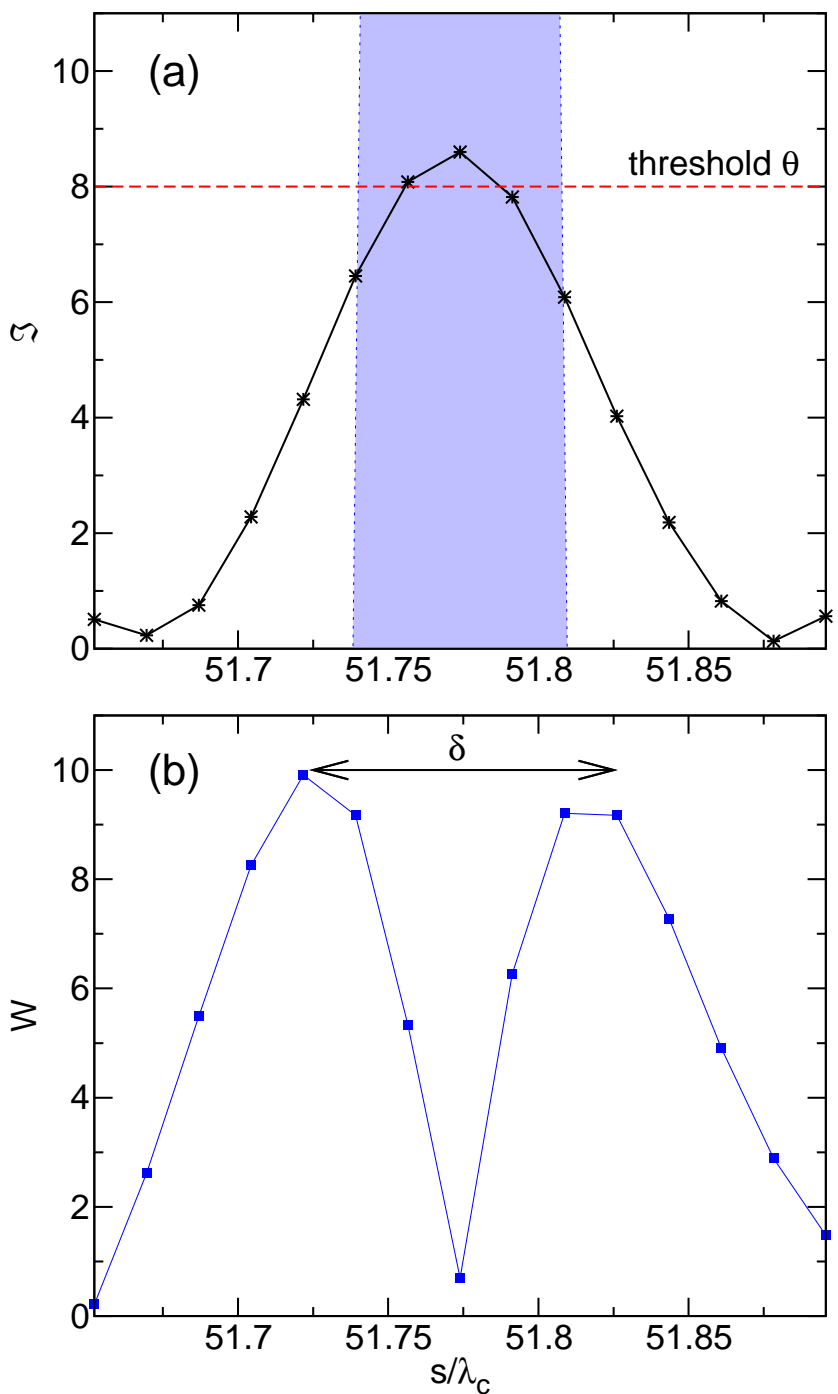

Figure 8. Tangential discontinuity captured with the PVI-technique, using $\Im\left(\Delta s=0.67 \lambda_{d}, \ell=\right.$ $535 \lambda_{c}$ ) and a threshold $\theta=8$. Panel (a): PVI signal (black lines and stars) and the threshold (red dashed line). Panel (b): $W$-field given by Eq. (8) (blue lines and squares). The shaded (blue) region in the top panel represents the reconnection zone (see Fig. 3). As can be seen from the bottom panel, the distance between the two peaks in $\mathrm{W}$ (namely $\delta$ ) is very close the size of the RS. Note that taking as $\delta$ the size of the signal that exceeds the PVI threshold can strongly underestimate (or overestimate) the real size of the RS. 

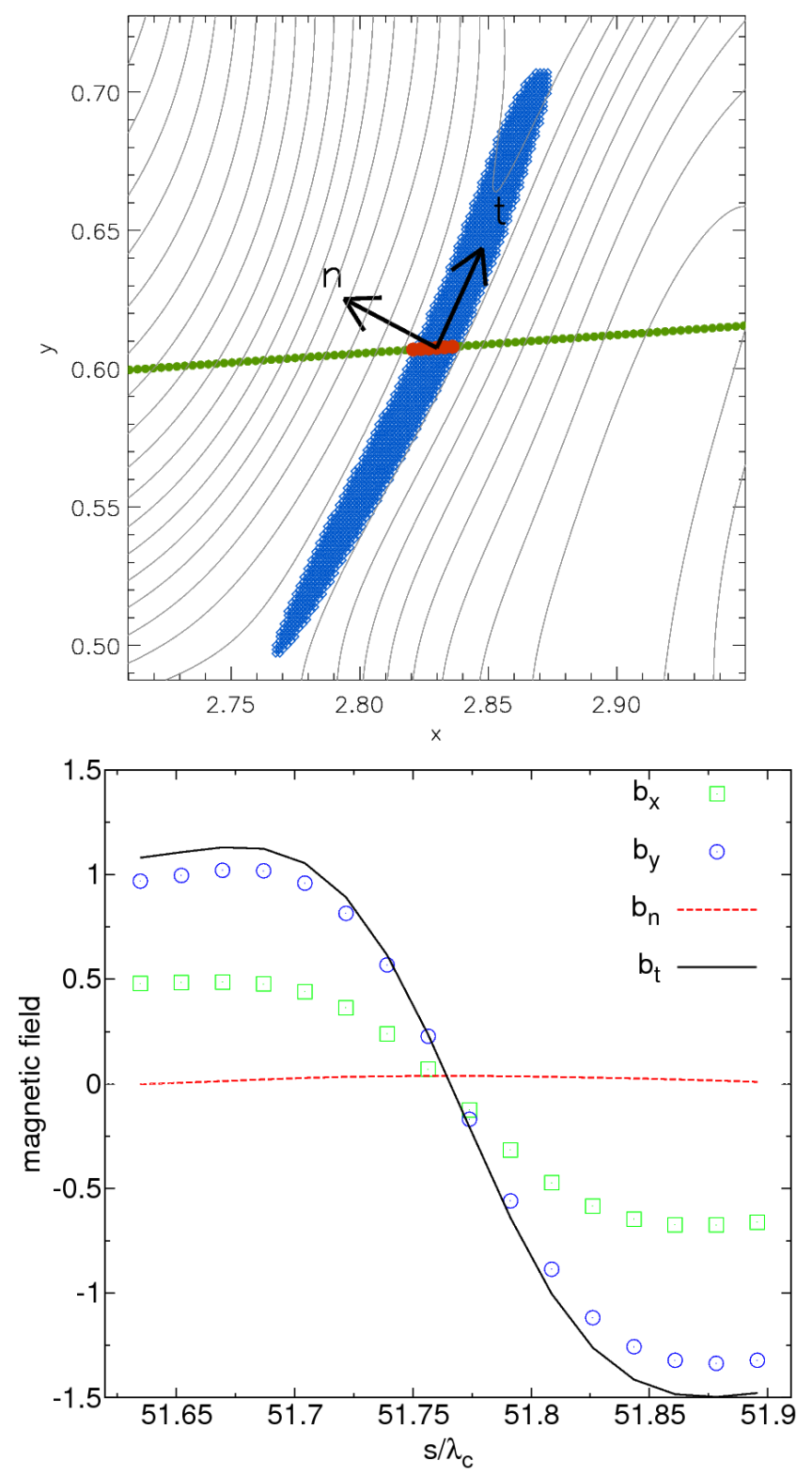

Figure 9. Zoom into the tangential discontinuity of Fig. 8. Top panel: two-dimensional crossing of the reconnection site (blue region, same as Fig. 3), together with the trajectory (green bullets), the TD captured by the PVI technique (red dots) and the minimum variance reference (MVA) frame $(\hat{\mathbf{n}}, \hat{\mathbf{t}})$ (black arrows). Bottom panel: magnetic field before the projection (open squares and circles) and in the minimum variance system (full and dashed lines). 


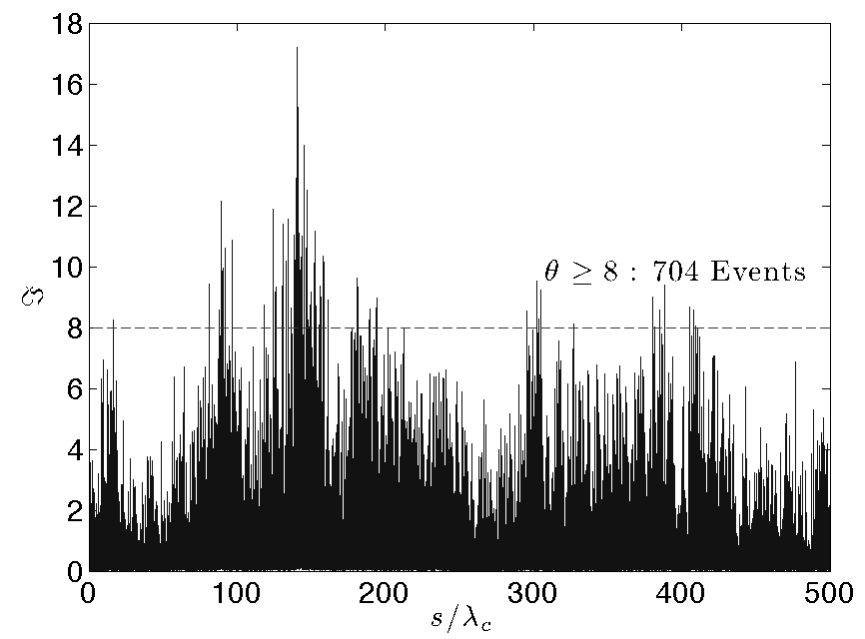

Figure 10. Spatial signal $\Im(\Delta s, \ell, s)$ (PVI) obtained from solar wind, with $\Delta s=20 \mathrm{~s}$ and $\ell \simeq 500 \lambda_{C}$

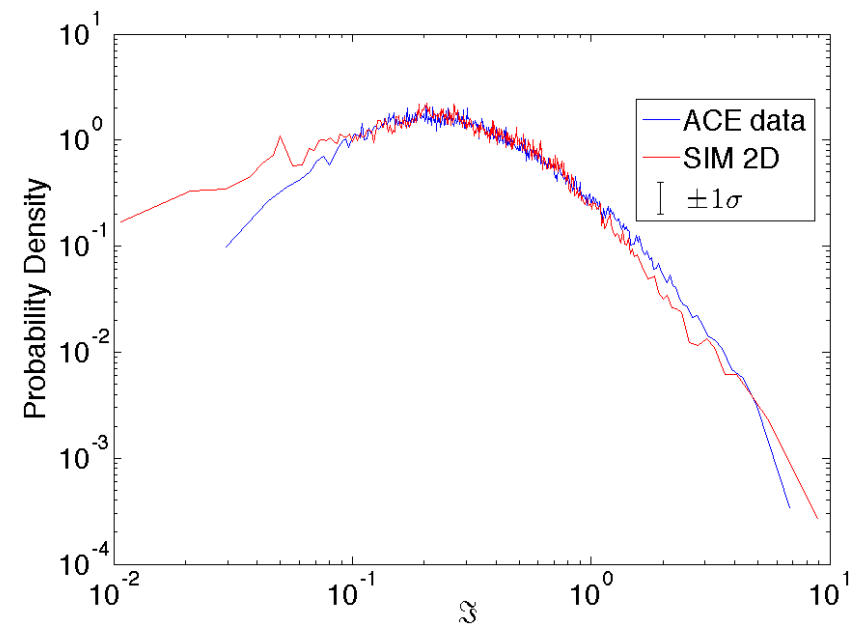

Figure 11. Probability density function of the spatial signal $\Im$ (PVI) obtained from ACE measurements (blue line) and simulation (red line). The error bar $\pm \sigma$ is displayed in the legend and the value of $\sigma$ is the expected fractional error in the PDF due to counting statistics. 